Sufficient has been said to indicate the value of this work to any physics library. There remains only one point on which the reviewer wishes to express his personal views. The author states in his preface: "In writing a book of this scope it is obvious that too much emphasis cannot be spared for style or for a detailed consideration as to the best organization in presenting the material. The need is for a critical compilation of the state of physical knowledge in the various fields to-day." One can appreciate the task of analysing and correlating the mass of data provided in this volume. But to the reader himself style and presentation of material are very important, and he may feel that had the author been in a position to spare the time, the volume could have been pruned without loss of conciseness and with some improvements in its arrangement. Again, the use of italics for purposes of emphasis is valuable. But when it is used fifty times in ten pages (pp. 419-428, taken at random) its effect on the reader may not be quite what the author intended.

\title{
AN INTRODUCTION TO MATHEMATICAL ANALYSIS
}

\section{Advanced Calculus}

By Dr. C. A. Stewart. Pp. xviii +523. (London : Methuen and Co., Ltd., 1940.) 258.

$\mathrm{W}$ ITH the ever-widening scope of modern mathematical analysis and its many ramifications, it is quite impossible to include, in a single volume of reasonable size, an adequate and exhaustive discussion of the calculus in its more advanced stages. It therefore becomes necessary, in planning a thoroughly sound course in the subject, to consider several important aspects of the vast field confronting a modern writer. The limitation of space renders the selection of subjectmatter fundamentally dependent upon the aim of the course, which may or may not be related to the content of specific examination syllabuses. Logical development, too, may lead to the inclusion of many topics which, at present, may only be of academic interest, while others, of greater practical value, may have to be omitted. The experience and training of the writer may also have, more or less, a bearing on both these considerations.

With such thoughts in mind, it is interesting to turn to Dr. Stewart's volume, in which a good course, especially useful to students reading for mathematical honours, is thoroughly well laid out. The author fully appreciates his difficulty in selecting from such a vast field for, in his preface, he states: "Although this book is intended for students who have already acquired some knowledge of the elements of the calculus, it must be regarded merely as an introduction to the more advanced parts of the subject."

Although the subject-matter of a first course is included, the earlier concepts, regarded as intuitively obvious in many cases, are later reexamined in order that they may be satisfactorily founded upon a rigorously valid basis. Space has, however, only permitted the author to deal with those fundamental theorems which are essential to the developments considered or are likely to be needed in important applications. The first five chapters are devoted to the usual rigorous course in calculus up to the integration of functions of one variable. Then follow chapters on Jacobians ; indeterminate forms, maxima and minima; vectors, twisted curves and tensors; multiple integrals ; functions of a complex variable, conformal representation and contour integration; infinite series, products, expansions, infinite integrals and non-convergent series, and finally, Bernoullian polynomials, gamma and beta functions, the formulæ of Binet and Gauss, and Dirichlet's integral. Only the simplest theorems relating to the theory of sets, Lebesgue integration, finite differences, tensors, one-one correspondence, analytic continuation, etc., have been given. Importance is rightly attached tó the approximate forms of functions, approximate integration and summation of series, but here again space has only allowed fundamental results to be dealt with.

From this very brief outline it will be seen that the scope of the course is wide. It has been skilfully planned and clearly presented, the diagrams deserving a special word of commendation. Many worked examples, often connected with important applications, are provided, and each chapter is supplied with appropriate exercises for the student's practice. Answers to these, where relevant, are also given.

It will be observed that no attempt has been made to include the theory of differential equations or of the functions that arise directly from them. It is hoped, however, that Dr. Stewart will consider the possibility of providing a companion volume devoted to this essential subject for, in the modern exploration of analysis, there is a tendency to overlook the supreme practical importance of this branch of mathematics, which really forms the very foundation of the higher stages of applied science. Such a volume would certainly be needed to supplement much of the present course by students reading for honours in science, notably physics. 